\title{
MMP13 Mutations are the Cause of Recessive Metaphyseal Dysplasia, Spahr Type
}

\section{Luisa Bonafé, ${ }^{1 *}$ Jinlong Liang, ${ }^{2}$ Maria W. Gorna, ${ }^{3}$ Qingyan Zhang, ${ }^{2}$ Russia Ha-Vinh, ${ }^{1}$} Ana Belinda Campos-Xavier, ${ }^{1}$ Sheila Unger, ${ }^{4}$ Jacques S. Beckmann, ${ }^{4}$ Antony Le Béchec, ${ }^{5}$ Brian Stevenson, ${ }^{5}$ Andres Giedion, ${ }^{6}$ Xuanzhu Liu, ${ }^{2}$ Giulio Superti-Furga, ${ }^{3}$ Wei Wang, ${ }^{2}$ André Spahr, ${ }^{7}$ and Andrea Superti-Furga ${ }^{1,8}$

\author{
${ }^{1}$ Division of Molecular Pediatrics, Lausanne University Hospital, Lausanne, Switzerland \\ ${ }^{2}$ BGI-Shenzhen, Shenzhen, China \\ ${ }^{3}$ CeMM Research Center for Molecular Medicine of the Austrian Academy of Sciences, Vienna, Austria \\ ${ }^{4}$ Medical Genetics Service, Lausanne University Hospital, Lausanne, Switzerland \\ ${ }^{5}$ Vital-IT-High Performance Computing Center, Swiss Institute of Bioinformatics, University of Lausanne, Lausanne, Switzerland \\ ${ }^{6}$ Division of Radiology, University Children's Hospital, Zurich, Switzerland \\ ${ }^{7}$ Cabinet de Pédiatrie, Sion, Switzerland \\ ${ }^{8}$ Department of Pediatrics, Lausanne University Hospital, University of Lausanne, Lausanne, Switzerland
}

Manuscript Received: 2 November 2013; Manuscript Accepted: 19 December 2013

Metaphyseal dysplasia, Spahr type (MDST; OMIM 250400) was described in 1961 based on the observation of four children in one family who had rickets-like metaphyseal changes but normal blood chemistry and moderate short stature. Its molecular basis and nosologic status remained unknown. We followed up on those individuals and diagnosed the disorder in an additional member of the family. We used exome sequencing to ascertain the underlying mutation and explored its consequences on threedimensional models of the affected protein. The MDST phenotype is associated with moderate short stature and knee pain in adults, while extra-skeletal complications are not observed. The sequencing showed that MDST segregated with a c.619T $>$ G single nucleotide transversion in MMP13. The predicted nonconservative amino acid substitution, p.Trp207Gly, disrupts a crucial hydrogen bond in the calcium-binding region of the catalytic domain of the matrix metalloproteinase, MMP13. The MDST phenotype is associated with recessive MMP13 mutations, confirming the importance of this metalloproteinase in the metaphyseal growth plate. Dominant MMP13 mutations have been associated with metaphyseal anadysplasia (OMIM 602111), while a single child homozygous for a MMP13 mutation had been previously diagnosed as "recessive metaphyseal anadysplasia," that we conclude is the same nosologic entity as MDST. Molecular confirmation of MDST allows distinction of it from dominant conditions (e.g., metaphyseal dysplasia, Schmid type; OMIM \# 156500 ) and from more severe multi-system conditions (such as cartilage-hair hypoplasia; OMIM \# 250250) and to give precise recurrence risks and prognosis. ๑ 2014 Wiley Periodicals, Inc.

Key words: metaphyseal dysplasia; Spahr dysplasia; MMP13; metalloproteinase; exome sequencing
How to Cite this Article:

Bonafé L, Liang J, Gorna M, Zhang Q, Ha-Vinh R, Campos-Xavier AB, Unger S, Beckmann J, Le Béchec A, Stevenson B, Giedion A, Liu X, Superti-Furga G, Wang W, Spahr A, Superti-Furga A. 2014. MMP13 mutations are the cause of recessive metaphyseal dysplasia, spahr type. Am J Med Genet Part A 9999:1-5.

Conflict of interest: none.

Luisa Bonafé, Jinlong Liang, and Maria Gorna contributed equally to this work.

Wei Wang, André Spahr, and Andrea Superti-Furga are the joint senior authors.

Grant sponsor: Swiss National Foundation; Grant number: 310030_132940; Grant sponsor: Leenaards Foundation (Lausanne); Grant sponsor: Faculty of Biology and Medicine of the Lausanne University (Fonds de Recherche en Pédiatrie).

*Correspondence to:

Professor Luisa Bonafé, M.D., Ph.D., Division of Molecular Pediatrics, Centre Hospitalier Universitaire Vaudois Av. Decker, 21011 Lausanne, Switzerland. E-mail: luisa.bonafe@chuv.ch Article first published online in Wiley Online Library (wileyonlinelibrary.com): 00 Month 2014 DOI 10.1002/ajmg.a.36431 


\section{${ }^{1}$ INTRODUCTION}

In 1961, André and Ines Spahr described a family from the Swiss Canton of Valais in which four of five siblings had moderate short stature, mild genua vara, and radiographic signs of metaphyseal dysplasia, but no biochemical signs of rickets [Spahr and SpahrHartmann, 1961]. Normal height parents and the high consanguinity rate in the village of origin of that family suggested a recessively inherited metaphyseal dysplasia. The condition became known as metaphyseal dysplasia, Spahr type (MDST) and was included in the OMIM catalog (OMIM 250400) as well as in the Nosology of Genetic Skeletal Disorders since 1970 [Warman et al., 2011]. It is very rare, with only two reports following the original one [Farag and Teebi, 1990; Megarbane et al., 2008]. Having access to the original family described in 1961, we set out to obtain a clinical follow-up as well as DNA samples from selected family members to explore its molecular basis.

\section{MATERIALS AND METHODS}

We studied DNA from the original family reported by Spahr and Spahr-Hartmann [1961] (Fig. 1). The study was conducted within the frame of a Swiss National Foundation-funded research project on chondrodysplasias for which ethical approval was obtained from the State Ethics Committee (Canton Vaud). Informed consent was obtained from affected individuals and their relatives, and blood was drawn into EDTA tubes and DNA extracted by standard procedures. In a first series of experiments, the exons and intron/exon boundaries of COL10A1 and RMRP (mutations in which cause metaphyseal chondrodysplasia, Schmid type (MCS) [Warman et al., 1993] and cartilage-hair hypoplasia (CHH) [Bonafe et al., 2002], respectively) were sequenced. Subsequently, the DNA of two affected (II-6, III-1) and one unaffected, obligate carrier individuals (I-3) were subjected to exome sequencing using pub- lished protocols [Shi et al., 2011]. The results were analyzed with an informatics pipeline excluding variants present at significant frequency in public databases, as well as variants frequently observed in the in-house database. Results were further prioritized in terms of data quality and, most significantly, assuming homozygosity by descent in the two affected individuals and heterozygosity in the obligate carrier. Candidate mutations were screened using known annotations for gene function and associated phenotypes.

Sanger sequencing was used to validate the presence and status of this mutation in the individuals included in the original exome sequencing study as well as in five other obligate carriers in the pedigree.

\section{RESULTS \\ Clinical Follow-up}

The original family comprised one unaffected and four affected sibs (Fig. 1). Of the four affected sibs, one was deceased because of an accident. The female Individual II-6 was followed up at age 47 years. Her adult height was $138 \mathrm{~cm}$, and her weight was $53 \mathrm{~kg}$. She had been professionally active as a secretary and had not founded a family. She was in good general health but reported recurrent knee pain. Magnetic resonance studies were reported to show significant knee arthritis with osteochondritis dissecans and presence of intraarticular free bodies. Her older brother, individual II-5, was reported to be $167 \mathrm{~cm}$ tall. He was professionally active, had married and had children. The younger brother, individual II-7, was reported to measure $150 \mathrm{~cm}$. He had not married. Neither reported chronic health problems.

Several years after the first report on MDST in 1961, we diagnosed the same disorder in a first cousin once removed of the original sibs (Individual III-1 in the pedigree, Fig. 1). This female individual, born in 1971, came to medical attention at age seven

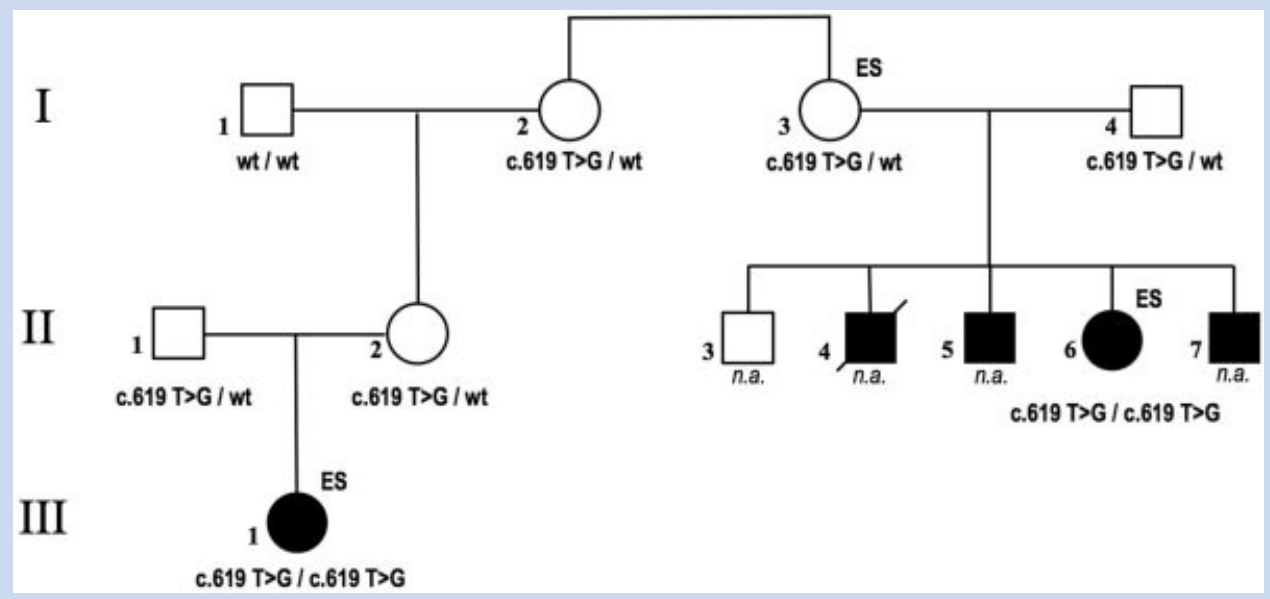

FIG. 1. Pedigree and sequence data from the family with MDST. Black symbols denote individuals diagnosed with MDST, white symbols denote clinically unaffected individuals. Individuals II-3 to II-7 correspond to the sibship described in the original paper. Individual III-1 was diagnosed subsequently. "ES" denotes the three individuals, whose DNA has been subjected to exome sequencing. The indications below the symbols indicate the results obtained by Sanger-type PCR sequencing of affected and unaffected individuals. N.A., sample not available. 
years because of short stature and intermittent knee pain. Her parents heights were $167 \mathrm{~cm}$ (father) and $155 \mathrm{~cm}$ (mother), giving her a target height of approximately $155 \mathrm{~cm}$. Her height at age 7 years was at $-2.5 \mathrm{SD}$, and her growth curve remained below the third centile, but parallel to it, until age 13 when she was lost to follow up. Radiographs at age 7 years showed metaphyseal dysplasia. When re-contacted at age 31 years, her height was $150 \mathrm{~cm}$ (thus only $5 \mathrm{~cm}$ below her genetic target height), her weight $54 \mathrm{~kg}$. She no longer complained of knee pain. She had trained and worked as a nurse and had one healthy child.

\section{Molecular Findings}

No mutation was found in the COL1OA1 and RMRP genes, first candidates for this metaphyseal dysplasia.

Upon inspection of the filtered data from exome sequencing, only one mutation met the criteria of correct segregation and low frequency on control datasets: a c.619T $>\mathrm{G}$ transversion which predicts p.Trp207Gly in the MMP13 protein (nucleotide 102824903 of chromosome 11, A $>$ C; Ensembl transcript ENST00000571284, MMP13, c.619T $>$ G). Sanger sequencing confirmed homozygosity in the two affected individuals, and heterozygosity in all obligate carriers (see pedigree in Fig. 1).
Residue Trp207 is highly conserved both across species and between different human MMPs (data not shown). The SIFT, POLYPHEN and PROVEAN programs predict the substitution p.Trp207Gly to be damaging (SIFT score: 0, POLYPHEN HumVar score: 1.00, PROVEAN score: -10.091). As fresh-frozen plasma or cultured cells for assessment of MMP13 activity were unavailable, we used molecular modeling to study the position and role of the affected residue, Trp207, within MMP13. The results are depicted in Figure 2. The catalytic site of MMP13 is lined by three histidine residues (His222, His226, and His232) that coordinate the zinc atom. Residue Trp207 is buried in the core of the catalytic domain, and its bulky hydrophobic character is likely necessary to maintain the proper architecture of the protein and to prevent misfolding. A glycine in the same position would not be able to fulfill the same role as the tryptophan due to lack of a side chain. In addition, the two hydrogen bonds formed by Trp207 support residues Asp202 and Asp128 that coordinate the structural calcium ions. A substitution to glycine would result in the loss of one of the hydrogen bonds, which would likely diminish the stability of the protein fold.

The c.619T $>$ G sequence change was absent in over 100 local control individuals. Interestingly, this change (that has no clinical consequences at heterozygosity, as the molecularly proven carriers in the family have a normal skeletal phenotype) accounted for two of approximately

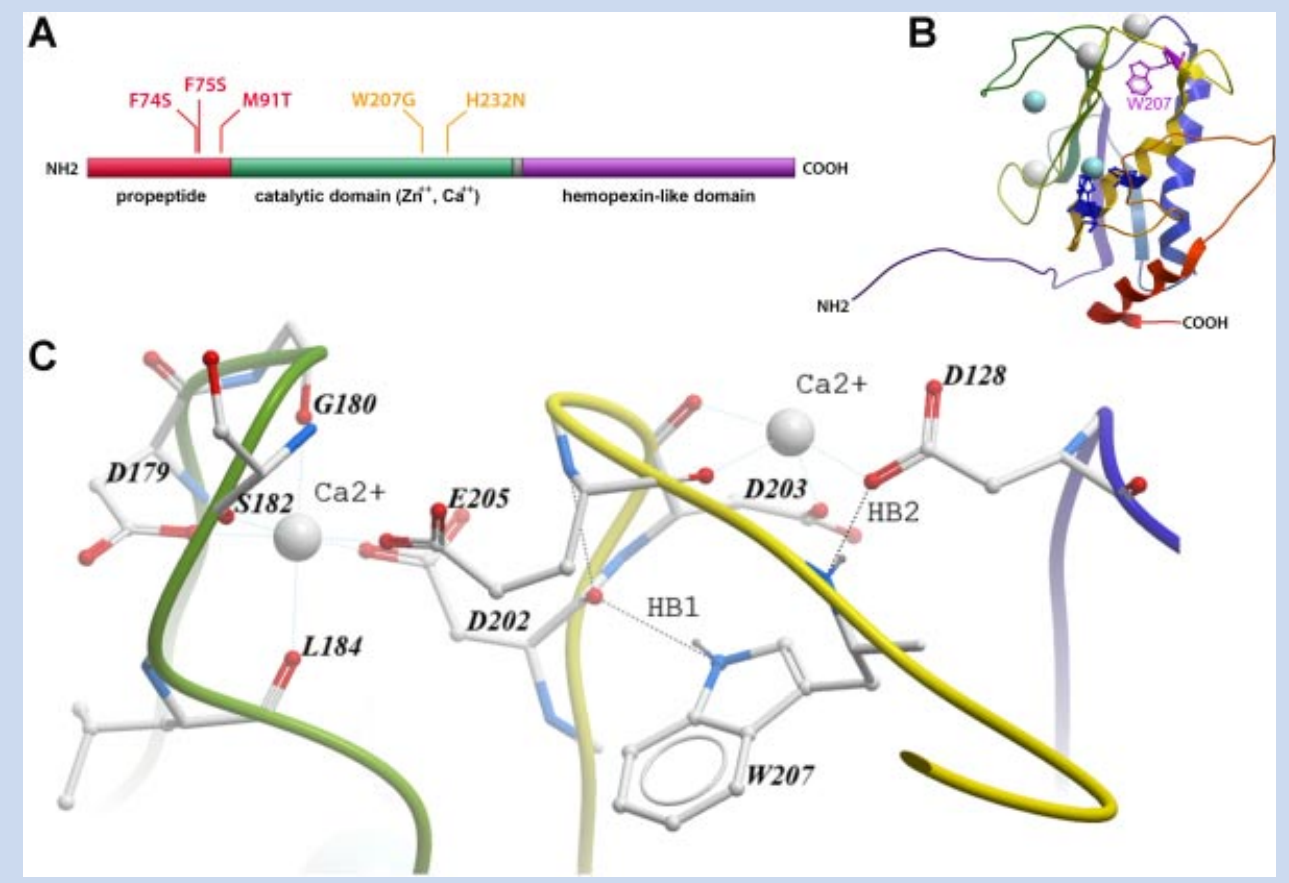

FIG. 2. Molecular pathology of MMP13. A: simplified scheme of the MMP13 protein with its domains. The dominant mutations (in red] are located in the propeptide domain. The recessive mutations (in orange) p.Trp207Gly, as found in the family reported here, and p.His232Asn, seen in a single patient, are in the catalytic domain. B: Crystal structure of MMP13 (PDB entry 3ZXH) in cartoon representation (the inhibitor present in the structure has been removed for simplicity). The mutated residue Trp207 (W207) is shown as purple sticks, and the three histidines (H222, H226, H232) coordinating the catalytic zinc ion are in dark blue. Zinc ions are depicted as cyan spheres, and calcium ions are white spheres. C: Close view on the Trp207 [W207) residue in the vicinity of two $\mathrm{Ca2}+$ binding sites. The side chain of W207 forms a hydrogen bond (black dotted line, HB1] with the carbonyl oxygen of D202, which coordinates one of the calcium ions. The main chain nitrogen of W207 forms a hydrogen bond (HB2) with D128, which coordinates another calcium ion. 
13,000 unselected alleles included in the Washington University Exome Variant Server (http://evs.gs.washington.edu/) cohort, giving it an allelic frequency of approximately 0.00015 ; such a frequency is in the range of a very rare recessive allele.

\section{DISCUSSION}

The exome analysis and the subsequent direct PCR sequencing study showed that the two affected individuals were homozygous and unaffected obligate carriers heterozygous for a single nucleotide transversion predicting a p.Trp207Gly substitution in MMP13. Even in absence of experimental functional data, the genetic evidence (segregation in the family and rarity in control alleles), the molecular modeling, and the prediction data strongly suggest that p.Trp207Gly is pathogenic in this family and not a harmless variant.

Mice in whom the mmp13 has been inactivated display a disturbed structure of the metaphyseal growth plate [Stickens et al., 2004], linking cartilage matrix degradation to the proliferation of chondrocytes at the growth plate. In humans, the majority of MMP13 mutations act as dominant negative and result in metaphyseal anadysplasia [Lausch et al., 2009]. The MMP13 protein has three main domains; the pro-domain, the catalytic domain, and the C-terminal domain with four hemopexin-like subdomains [Leeman et al., 2002] (see http://www.uniprot.org/uniprot/P45452 for details). The more common MMP13 mutations associated with dominant metaphyseal anadysplasia [Lausch et al., 2009], as well as a further dominant mutation associated with spondylo-metaphyseal dysplasia [Kennedy et al., 2005], are located in the propeptide domain (Fig. 2A). By disrupting the tertiary structure of the propeptide domain, they result in premature activation of the proteinase, which in turn determines autocatalytic self-inactivation of MMP13 as well as in heterocatalytic inactivation of the related enzyme, MMP9 [Lausch et al., 2009]. The catalytic region of MMP13 is situated in the central portion of the molecule: amino acids 172 to 232 include all calcium-binding and zinc-binding residues necessary for catalysis (Fig. 2A). In the previous study, we had observed a child who was homozygous for a mutation in MMP13 and we had given him the diagnosis of recessive metaphyseal anadysplasia [Lausch et al., 2009]. The MMP13 mutation in that child, p.His232Asn, affected the histidine residue at position 232 involved in zinc binding [Lausch et al., 2009]. The novel mutation segregating in the present MDST family, p.Trp207Gly, affects a residue involved in the stabilization of the calcium-binding region (Fig. 2B and C). Together, the data suggest that the phenotype of MDST is associated with homozygosity for a loss of function mutation in MMP13 and that the one child with "recessive metaphyseal anadysplasia" can be retrospectively diagnosed as MDST. As we failed to recognize this phenotypic similarity, we did not analyze MMP13 and MMP9 directly, but took the more complex strategy of exome sequencing. On the positive side, the exome approach is unbiased and adds significance to our findings.

Although the follow-up of affected individuals in this and in the previous study [Lausch et al., 2009] was limited, we conclude that MDST is associated with short stature of variable degree: the shortest affected patient was II-6, a female with a final height of $138 \mathrm{~cm}$, and the tallest individuals was II-5, reported to be $167 \mathrm{~cm}$ tall. Individual II-6 also suffered from knee joint degeneration, while Patient III-1 had had knee pain in childhood but was free from symptoms in adulthood. Otherwise, no other significant symptom or clinical sign was reported. We would also conclude that MDST is possibly, but not consistently, associated with knee pain in childhood and/or early-onset degenerative arthritis of the knee, although the latter finding may be coincidental as it was present in only one of the five adult individuals.

The prognostic implications are quite different in the different conditions with metaphyseal dysplasia, for example, MCS [Warman et al., 1993] (OMIM \# 156500), CHH [Bonafe et al., 2005] (OMIM \# 250250), or dominant metaphyseal anadysplasia (MAD; OMIM \# 602111). Are there criteria that allow for a differentiation between these disorders? The clinical presentation of MDST is similar to that of MCS, with normal length at birth, bowing and shortening of the legs becoming apparent after the child begins to stand, and a waddling gait. In contrast, patients with $\mathrm{CHH}$ are usually short at birth or display growth failure already in the first months of life; their adult height is significantly shorter than in the two other conditions. Patients with $\mathrm{CHH}$ also present with varus deformity of the lower limbs but this is usually less pronounced than in patients with MCS or MDST and waddling gait is rare. The main distinctive clinical features of patients with $\mathrm{CHH}$ are short hands and feet and joint laxity, which are not found in the two other conditions. The presence of sparse hair and immune deficiency or anemia are not obligate diagnostic criteria of $\mathrm{CHH}$ as about $30 \%$ of patients present only the skeletal features of the disease. However, even in the "isolated skeletal variant" of $\mathrm{CHH}$ [Bonafe et al., 2002] there seems to be a persistent risk of hematopoietic cancer at young adult age, which is not the case for patients with MDST or MCS. The facial appearance is normal in all three conditions.

Radiographic features include metaphyseal dysplasia mostly prominent at the lower limbs in all three conditions; while the distal femoral and proximal tibia metaphyses are involved in all three, the varus deformity typical for MCS is absent or less pronounced in MDST. In $\mathrm{CHH}$, the femoral neck is often constricted and distinct from MDST and MCS. The capital femoral epiphyses are rather large in MCS during childhood, whereas they are of small size in MDST. The radiographic appearance of spine and pelvis is normal in all three conditions although mild platyspondyly may be seen occasionally in MCS. The differentiation of MDST and MAD may be difficult, as the conditions are almost superimposable, the main difference being in stature: while MDST is associated with short stature in childhood and in adulthood, the height of individuals affected by the dominantly inherited MAD is usually between -1 and -2 SD. Finally, spondyloepi-metaphyseal dysplasia (SEMD) Missouri type is a condition reported in a single family associated with a dominant mutations (p.Phe56Ser) in MMP13 located in the prodomain of the protein; the metaphyseal involvement has been reported in combination with a mild degree of vertebral involvement [Kennedy et al., 2005]. However, a very similar mutation (p.Phe55Ser vs. p.Phe56Ser) has been repeatedly observed in dominant $\mathrm{MAD}$, and the biochemical consequences of the two mutations appear to be the same [Lausch et al., 2009], suggesting that in practical terms, the two conditions are indistinguishable. In summary, MDST, a recessive entity, should be readily distinguishable from $\mathrm{CHH}$, while distinction from MCD and from MAD would require either the presence of a dominant pedigree, or molecular genetic testing. 
Besides unraveling the molecular basis of a disorder described more than 50 years ago and filling a gap in the Nosology of genetic bone disease [Warman et al., 2011], the results demonstrate that the observation of metaphyseal dysplasia in an infant and small child requires careful differential diagnosis. With next generation sequencing rapidly entering the diagnostic scene, the availability of documented genotype-phenotype correlations, such as the one presented here, will be of crucial importance in providing families and physicians with information on diagnosis and prognosis.

\section{ACKNOWLEDGMENTS}

We are thankful to the members of the original family for their willingness to provide information and blood samples for this study, and to Mrs. Carole Chiesa and Sonia Do Vale-Pereira for technical assistance. The study was supported by the Swiss National Foundation (grant 310030_132940 to L.B.), by the Leenaards Foundation (Lausanne) and by the Faculty of Biology and Medicine of the Lausanne University (Fonds de Recherche en Pédiatrie).This paper is dedicated to the memory of our mentor and friend Professor Andres Giedion (1925-2013).

\section{WEB RESOURCES}

UniProt, http://www.uniprot.org/uniprot/P45452

The National Heart Lung and Blood Institute (NHLBI) Exome

Sequencing Project, http://evs.gs.washington.edu/EVS/

Online Mendelian Inheritance in Man (OMIM), http://www. omim.org/

PolyPhen-2, http://genetics.bwh.harvard.edu/pph2/index.shtml

Protein Variation Effect Analyzer (PROVEAN), http://provean. jcvi.org/index.php

SIFT, http://sift.jcvi.org/

\section{REFERENCES}

Bonafe L, Schmitt K, Eich G, Giedion A, Superti-Furga A. 2002. RMRP gene sequence analysis confirms a cartilage-hair hypoplasia variant with only skeletal manifestations and reveals a high density of single-nucleotide polymorphisms. Clin Genet 61:146-151.
Bonafe L, Dermitzakis ET, Unger S, Campos-Xavier BA, Zankl A, Ucla C, Antonarakis SE, Superti-Furga A, Reymond A. 2005. Evolutionary comparison provides evidence for pathogenicity of RMRP mutations. PLoS Genet 1:e47.

Farag TI, Teebi AS. 1990. The second family with Spahr-type metaphyseal chondrodysplasia: Autosomal recessive inheritance confirmed. Clin Genet 38:237-239.

Kennedy AM, Inada M, Krane SM, Christie PT, Harding B, López-Otín C, Sánchez LM, Pannett AA, Dearlove A, Hartley C, Byrne MH, Reed AA, Nesbit MA, Whyte MP, Thakker RV. 2005. MMP13 mutation causes spondyloepimetaphyseal dysplasia, Missouri type (SEMD(MO). J Clin Invest 115:2832-2842.

Lausch E, Keppler R, Hilbert K, Cormier-Daire V, Nikkel S, Nishimura G, Unger S, Spranger J, Superti-Furga A, Zabel B. 2009. Mutations in MMP9 and MMP13 determine the mode of inheritance and the clinical spectrum of metaphyseal anadysplasia. Am J Hum Genet 85:168-178.

Leeman MF, Curran S, Murray GI. 2002. The structure, regulation, and function of human matrix metalloproteinase-13. Crit Rev Biochem Mol Biol 37:149-166.

Megarbane A, Chouery E, Ghanem I. 2008. A multiplex family with possible metaphyseal Spahr-type dysplasia and exclusion of RMRP and COL10A1 as candidate genes. Am J Med Genet A 146A:1865-1870.

Shi Y, Li Y, Zhang D, Zhang H, Li Y, Lu F, Liu X, He F, Gong B, Cai L, Li R, Liao S, Ma S, Lin H, Cheng J, Zheng H, Shan Y, Chen B, Hu J, Jin X, Zhao P, Chen Y, Zhang Y, Lin Y, Li X, Fan Y, Yang H, Wang J, Yang Z. 2011. Exome sequencing identifies ZNF644 mutations in high myopia. PLoS Genet 7:e1002084.

Spahr A, Spahr-Hartmann I. 1961. Dysostose metaphysaire familiale. Etude de 4 cas dans une fratrie. Familial metaphysial dysostosis. Study of 4 cases in siblings. Helv Paediatr Acta 16:836-849.

Stickens D, Behonick DJ, Ortega N, Heyer B, Hartenstein B, Yu Y, Fosang AJ, Schorpp-Kistner M, Angel P, Werb Z. 2004. Altered endochondral bone development in matrix metalloproteinase 13-deficient mice. Development 131:5883-5895.

Warman ML, Abbott M, Apte SS, Hefferon T, McIntosh I, Cohn DH, Hecht JT, Olsen BR, Francomano CA. 1993. A type X collagen mutation causes Schmid metaphyseal chondrodysplasia. Nat Genet 5:79-82.

Warman ML, Cormier-Daire V, Hall C, Krakow D, Lachman R, LeMerrer M, Mortier G, Mundlos S, Nishimura G, Rimoin DL, Robertson S, Savarirayan R, Sillence D, Spranger J, Unger S, Zabel B, Superti-Furga A. 2011. Nosology and classification of genetic skeletal disorders: 2010 Revision. Am J Med Genet A 155A:943-968. 